\title{
Molecular understanding of peripheral T-cell lymphomas, not otherwise specified (PTCL, NOS): A complex disease category
}

\author{
Mamiko Sakata-Yanagimoto, ${ }^{1,2,3)}$ Kota Fukumoto, ${ }^{2,3)}$ Kennosuke Karube, ${ }^{4)}$ Shigeru Chiba ${ }^{1,2,3)}$
}

Peripheral T-cell lymphoma, not otherwise specified (PTCL, NOS) includes various diseases. Attempts have been made to identify distinct properties of disease within the PTCL, NOS classification and evaluate their significance to prognosis. Comprehensive gene expression analysis and evaluation of genomic abnormalities have successfully identified specific diseases from heterogeneous PTCL, NOS cases. For example, cases with properties of T follicular helper cells have been identified and classified as an entity resembling angioimmunoblastic T-cell lymphoma (AITL), based on both immunohistochemistry and genomic features. Here, we focus on the molecular pathology of PTCL, NOS and discuss recent changes relevant to its classification.

Keywords: PTCL, nos, T follicular helper, AITL

\section{INTRODUCTION}

Peripheral T-cell lymphoma, not otherwise specified (PTCL, NOS) falls into the category of mature T/NK-cell neoplasms and accounts for $25.9 \%$ of total T/NK-cell neoplasms, according to the international peripheral T-cell lymphoma project (I-PTCL) published in 2008. ${ }^{1}$ Based on the original definition, PTCL, NOS is a mixed category: ${ }^{2}$ Patients are classified as PTCL, NOS when tumor tissues are infiltrated by atypical cells with properties of mature $\mathrm{T}$ cells but cannot be classified as any other mature T/NK-cell neoplasm. Recent analysis of gene expression profiles (GEPs) and genetic abnormalities in PTCL, NOS samples have identified novel diseases that fall into the PTCL, NOS category in PTCL, NOS. Better understanding of molecular pathways perturbed in mature T/NK-cell neoplasms, including PTCL, NOS, is now allowing us to distinguish among them more clearly in clinical settings. Below, we will review molecular understanding from the perspective of gene expression analysis and genomic abnormalities in PTCL, NOS.

\section{BOUNDARIES BETWEEN PTCL, NOS AND OTHER MATURE T/NK-CELL NEOPLASMS}

To clarify comparisons between morphological diagnosis and molecular classification, Iqbal et al. analyzed a series of GEPs as a study of the Lymphoma Leukemia Molecular Profiling Project (LLMPP) and I-PTCL. ${ }^{3}$ That analysis included 372 cases of mature T/NK-cell neoplasms of various subtypes, some previously characterized. ${ }^{4-8}$ For anaplastic large cell lymphoma (ALCL), ALK-positive, which is marked by an $A L K$ fusion gene, ${ }^{9}$ there was substantial agreement between pathological diagnosis and molecular classification, likely because ALK positivity clearly distinguishes this disease from others, and ALK tyrosine kinase induces an easily recognizable molecular signature. ${ }^{10}$ By contrast, there was not sufficient concordance between pathological and molecular classifications of PTCL, NOS and AITL: 21 of 150 (14\%) cases morphologically diagnosed with PTCL, NOS were reclassified as AITL based on molecular classification, while 26/117 (22\%) morphologically diagnosed as AITL were judged to be PTCL, NOS based on molecular classification. ${ }^{3}$ This discrepancy may be due in part to the fact that differences between AITL and PTCL, NOS were ambiguous

\footnotetext{
Received: November 13, 2020. Revised: December 30, 2020. Accepted: January 15, 2021. J-STAGE Advance Published: March 15, 2021

DOI: $10.3960 /$ jslrt.20059

${ }^{1}$ Department of Hematology, Faculty of Medicine, University of Tsukuba, Tsukuba, Japan, ${ }^{2}$ Department of Hematology, University of Tsukuba Hospital, Tsukuba, Japan, ${ }^{3)}$ Department of Hematology, Comprehensive Human Biosciences, University of Tsukuba, Tsukuba, Japan, ${ }^{4}$ Department of Pathology and Cell Biology, Graduate School of Medicine and Faculty of Medicine, University of the Ryukyus, Nishihara, Japan

Corresponding author: Mamiko Sakata-Yanagimoto, MD, PhD, Department of Hematology, Faculty of Medicine, University of Tsukuba, 1-1-1 Tennnodai, Tsukuba, Ibaraki 3050816, Japan. E-mail: sakatama-tky@umin.net

Copyright (C) 2021 The Japanese Society for Lymphoreticular Tissue Research

(cc)BY-NC-SA This work is licensed under a Creative Commons Attribution-NonCommercial-ShareAlike 4.0 International License.
} 
in 2014, when this work was published. ${ }^{3}$ It remains unclear whether concordance between morphological diagnosis and molecular classification will improve once the concept of nodal peripheral $\mathrm{T}$-cell lymphomas with $\mathrm{T}$ follicular helper phenotype (nPTCL-Tfh) noted below (section III, "PTCL, NOS subclassification by "cell of origin" of tumor cells") is established. $^{2}$ Similarly, 17/150 (11\%) cases of morphological PTCL, NOS cases exhibited molecular features of ALCL, ALK-negative, although all the cases morphologically diagnosed as ALCL were moleuclaly diagnosed as ALCL, ALK negative, ${ }^{3}$ suggesting that the boundary between PTCL, NOS and ALCL, ALK-negative, is ambiguous.

In a related study, Piccaluga et al. established molecular classifiers using GEPs from 244 PTCL cases with accuracy of $98 \%$ for AITL and $98 \%$ for ALC, ALK negative. ${ }^{11}$ An analysis of additional 132 previously published PTCL cases ${ }^{4-6,12}$ as a validation set showed the similar tendencies with accuracy of $77 \%$ for AITL and $93 \%$ for ALCL, ALK-negative. ${ }^{11}$

Proteins having cytotoxic functions such as TIA-1, granzyme B, and perforin are expressed in $41 \%{ }^{13}$ or $19.5 \%{ }^{14}$ of PTCL, NOS. Phenotypes seen in these cases overlapped with some organ-specific PTCLs, including enteropathyassociated T-cell lymphoma (EATL), hepatosplenic T-cell lymphoma (HSTL), and panniculitis-like T-cell lymphoma (SPTCL). Molecularly, SETD2 and STAT5B, the most frequently $(\sim 30 \%)$ mutated genes in $\mathrm{HSTL}^{15}$ and EATL, ${ }^{16}$ were rarely mutated in PTCL, NOS. ${ }^{17,18}$ SPTCL is characterized by germline HAVCR 2 mutations not identified in other hematopoietic neoplasms, including PTCL, NOS. ${ }^{19,20}$ These findings suggest that lymphomagenesis differs between PTCL, NOS and organ-specific PTCLs. Adult T-cell leukemia/lymphoma (ATLL), another T-cell lymphoma, is a separate disease entity caused by Human T-cell leukemia virus type 1. Despite this etiological difference, array-comparative genomic hybridization (CGH) identified a subset of PTCL, NOS that resembles ATLL. ${ }^{21}$ PTCL, NOS and ATLL also share numerous mutations including in genes associated with TCR-signaling. ${ }^{18,22}$

\section{PTCL, NOS SUBCLASSIFICATION BY "CELL OF ORIGIN" OF TUMOR CELIS (Table 1)}

Physiologically, T cells are classified into subtypes, depending on their function in the immune response, the profiles of cytokines they produce, or their immunophenotypes. PTCL is subdivided based on how well tumor cells correspond to certain subtypes of $\mathrm{T}$ cells, their so-called "normal counterparts". "Normal counterparts" are currently viewed as a tumor cell's "cell of origin". 2,23 However, "origin" may not indicate the precise beginning of tumorigenesis, based on analyses of mutation distribution in PTCL patients. ${ }^{24-27}$ Those studies suggest that the first hit may occur in immature hematopoietic stem cells residing in bone marrow, ${ }^{28}$ although tumor cells exhibit properties of mature T cells ${ }^{4}$ (See section IV, "Genomic abnormalities of PTCL, NOS"). This theory of PTCL origin is analogous to the proposal that first hits, such as $I g H / B C L 2$ translocation in follicular lymphoma ${ }^{29}$ or
$I g H / C C N D 1$ in mantle cell lymphoma, ${ }^{30}$ occur in pre-B cells in bone marrow, although these lymphoma cells resemble mature B cells.

An example of classifying a disease based on equating the "cell of origin" to a "normal counterpart" is that some PTCL, NOS cases diagnosed according to the 2008 WHO classification were shown to have characteristics of Tfh cells in tumor cells based on immunohistochemistry (IHC) $)^{31}$ but were later shown to share genetic features with AITL. ${ }^{32-35}$ These cases were subsequently defined as nPTCL-Tfh in a recently revised 2017 WHO classification and integrated into the provisional category, "AITL and other nodal lymphomas of Tfh cell origin". ${ }^{2}$ Tumor cells in this category, which includes AITL, nPTCL-Tfh and follicular T-cell lymphoma (FTCL), must express at least 2 (preferably 3 ) of the following Tfh markers based on IHC: CD10, BCL6 transcription repressor (BCL6), programmed cell death 1 (PDCD1/CD279/ PD-1), C-X-C motif chemokine ligand 13 (CXCL13), C-X-C motif chemokine receptor 5 (CXCR5), inducible $\mathrm{T}$ cell costimulatory (ICOS), and signaling lymphocytic activation molecule-associated protein (SAP). ${ }^{2}$

Iqbal et al. proposed to further classify PTCL, NOS based on GEPs ${ }^{2}$ as follows: PTCL, NOS with type1 helper T cell (Th1) characteristics expressing T-bet (TBX21) and its targets as PTCL-TBX21 (49\% of PTCL, NOS); PTCL, NOS with Th2 characteristics and expressing GATA binding protein 3 (GATA3) and its targets as PTCL-GATA3 (33\%); and PTCL, NOS with neither features as "unclassifiable" (18\%). Patients in the PTCL-GATA3 subgroup exhibited poorer prognosis than those in the PTCL-TBX21 subgroup. ${ }^{3}$ Wang et al. independently reported that GATA3 expression distinguished a PTCL, NOS subgroup with distinct molecular signatures, Th2 characteristics and poor prognosis. ${ }^{36}$ An aggressive clinical course in the PTCL-GATA3 subgroup is presumably due to higher activity of oncogenic pathways and more complex genomic abnormalities in that group relative to PTCL-TBX2117 (See section IV, "Genomic abnormalities of PTCL, NOS").

TBX21, GATA3, and BCL6 encode transcription factors near the top of the hierarchy regulating cell-fate decisions towards Th1, Th2, ${ }^{37}$ and $\mathrm{Tfh}^{38}$ cells, respectively. These factors activate transcriptional programs necessary for cell differentiation and expression of cytokines, chemokines, and their receptors - among them, interferon gamma (IFN $\gamma$ ), interleukin 2 receptor beta (IL2R $\beta$ ), c-c motif ligand 3 (CCL3), and CXCR3 for Th1 cells; IL4, IL13 and CCR4 for Th2 cells; and IL21, CXCL13, and CXCR5 for Tfh cells. Based on their physiological function, these transcription factors may directly activate oncogenic signaling in PTCL with skewed differentiation towards respective subtypes of helper T cells.

Amador et al. asked if "cell of origin" classifications, initially proposed by GEPs, could be reproduced by IHC in paraffin sections using antibodies for GATA3 and TBX21, and their target proteins CCR 4 and CXCR3. ${ }^{39}$ Percent positivity of tumor cells by immunochemistry and $\log 2$ values of transcript levels of GATA3 and CCR4, markers of PTCL-GATA3, 


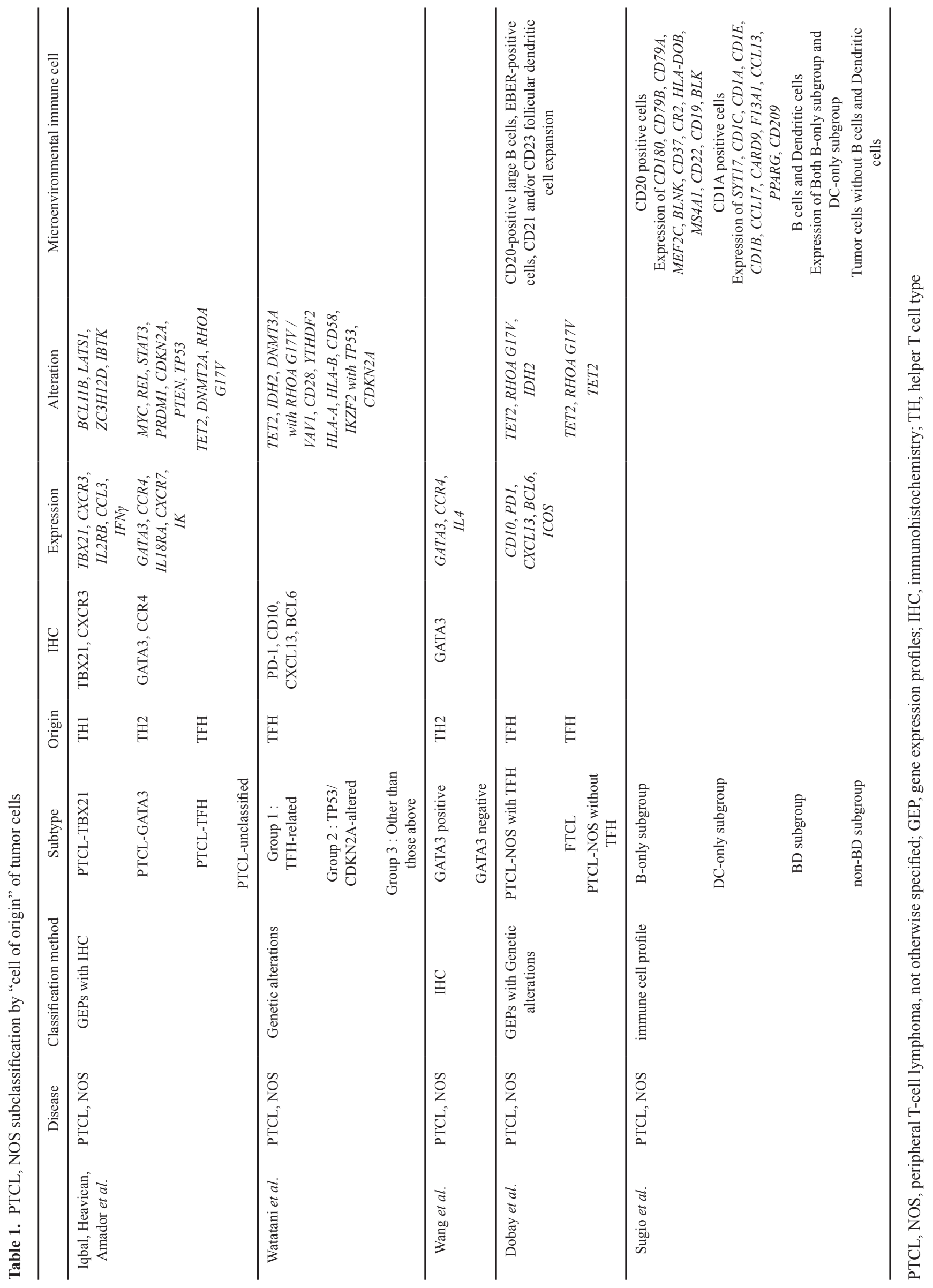


were highly correlated in a linear manner, while those of TBX21 and CXCR3 exhibited a curvilinear relationship. As a result, an IHC algorithm was established to diagnose PTCL-GATA3 versus PTCL-TBX21 among PTCL, NOS samples, enabling use of molecular classification in a clinical setting. ${ }^{39}$

\section{MICROENVIRONMENT OF PTCL, NOS}

PTCL, NOS tumor tissues are infiltrated with immune cells and are heterogeneous in their immune cell profiles (ICPs). Sugio et al. estimated ICPs from GEP data of 120 genes using digital count gene expression analysis of 68 PTCL, NOS cases and assessed their relationship to prognosis. ${ }^{40}$ Intriguingly, PTCL, NOS were classified as exhibiting characteristics of (i) B cells (B-only subgroup), (ii) dendritic cells (DC-only subgroup), (iii) both of these (BD subgroup), or (iv) none of these (non-BD subgroup). The non-BD subgroup had particularly poor prognosis, and macrophages expressing high levels of immune checkpoint molecules (such as programmed death ligand $1 / 2$ and indoleamine 2, 3-dioxygenase 1) were enriched in half of the non-BD subgroup. In contrast, patients in the BD subgroup had a more favorable prognosis.

Considering that, like their normal counterparts, tumor cells can produce cytokines and chemokines that promote migration and infiltration of immune cells expressing the corresponding receptors, the relationship between "cell of origin" classification and ICP is an intriguing topic of investigation. Amador et al. reported that most PTCL-TBX21 specimens exhibit a polymorphous appearance and harbor various types of inflammatory cells, while a more uniform expansion of tumor cells is seen in most PTCL-GATA3 cases. ${ }^{39}$ Wang et al. has observed infiltration of alternatively polarized macrophages expressing CD163 and phosphoSTAT3 (pSTAT3) induced by Th2-associated cytokines and IL-10 in a subset of PTCL. ${ }^{36}$ Although GATA3 functions in induction of these cytokines, GATA3 expression was not statistically correlated with $\mathrm{pSTAT} 3^{+} / \mathrm{CD} 163^{+}$macrophages in PTCL. ${ }^{36}$ ICPs in nPTCL-Tfh also remain to be elucidated: it is particularly critical to define the border between nPTCLTfh and AITL, the latter marked not only by prominent infiltration of inflammatory cells with a blood lineage but also by high endothelial venules and follicular dendritic cells. ${ }^{2}$

\section{GENOMIC ABNORMALITIES IN PTCL, NOS}

Since the human genome was sequenced in 2001, great progress has been made in analyzing the cancer genome. Accordingly, there are several reports of genomic abnormalities in mature T/NK-cell neoplasms, including PTCL, NOS, although many of the latter were first analyzed alongside AITL studies. Mutations found in mature T/NK-cell neoplasms include: (i) those widely seen in various cancers (TP53 and CDKN2A mutations/deletions), (ii) those widely seen in hematologic cancers (TET2, DNMT3A, and IDH2 mutations), (iii) those found in many subtypes of mature T/
NK-cell neoplasms (VAV1, PLCG1, and STAT3 mutations), and (iv) those specific to various subtypes of mature T/ NK-cell neoplasms (such as the p.Gly17Val RHOA mutation in AITL, nPTCL and FTCL, and $A L K$ translocations in ALCL, ALK-positive).

It is difficult to understand genomic abnormalities in PTCL, NOS, because historically most studies included nPTCL-Th in PTCL, NOS, although the 2017 WHO classification proposed moving nPTCL-Tfh from the category of PTCL, NOS to "AITL and other nodal lymphomas of Tfh cell origin". Some investigations have described mutation frequencies in PTCL, NOS with regards to the Tfh phenotype, while the definitions varies among studies. ${ }^{18,32-35}$ Lemonnier et al..$^{32}$ designated "TFH-like" PTCL, NOS as follows: “...within PTCL, NOS, a group without typical morphology of AITL but expressing Th follicular (TFH) cell markers (ie, PD-1, BCL6, and/or CXCL13) and/or having some other features reminiscent of AITL (ie, the presence of 2 of the following criteria: CD20-positive large B cells, EBVencoded small RNAs (EBER)-positive cells, CD21 and/or CD23 follicular dendritic cell expansion, or CD10 expression)". A paper by Vallois et $a l .{ }^{34}$ followed also this criteria. However, a study by Sakata-Yanagimoto et al..$^{33}$ defined TFH-like PTCL, NOS as follows: “...within PTCL, NOS cases, a subgroup without the typical morphology of AITL but having two or more of the following immunostaining features (i) positive staining for CD10 in tumor cells, (ii) positive staining for PD-1 in tumor cells, (iii) proliferation of CD21-positive follicular dendritic cells and (iv) the presence of EBER-positive B cells". Dobay et al. referred to the 2016 WHO classification criteria ${ }^{41}$ to diagnose TFH-like PTCL, but among seven TFH markers, five of them, including ICOS, PD-1, CXCL13, BCL6 and CD10, were examined. Watatani et al..$^{18}$ examined four TFH markers, including PD-1, CD10, CXCL13, and BCL6, and diagnosed PTCL, NOS cases as "TFH PTCL, NOS" if they expressed at least two TFH markers. Although the definition of "Tfh phenotype" in all of these papers differs from the current definition of nPTCL-Tfh, ${ }^{2}$ we refer to these PTCL, NOS with TFH features with various definitions as nPTCL-Tfh in this review.

\section{Genomic abnormalities and "cell of origin" classification in PTCL, NOS}

nPTCL-Tfh is predicted to exhibit genomic abnormalities similar to AITL based on studies of PTCL harboring Tfh features, ${ }^{32-35}$ although the definition of the diseases in these studies was not completely concordant with the current definition of nPTCL-Tfh. Hallmarks of nPTCL-Tfh include recurrent mutations in genes encoding epigenetic regulators (TET2 and $D N M T 3 A$ ), in p.Gly17Val RHOA, and in genes functioning in TCR signaling (CD28, PLCG1, and VAV1). Heavican et al. performed copy number analysis (CNA) in 69 cases of PTCL, NOS after exclusion of nPTCL-Tfh together with targeted sequencing of 334 genes in 31 cases and found that the PTCL-GATA3 subgroup exhibited more complex genomic abnormalities than did PTCL-TBX21: Frequent genomic amplifications of loci encoding the protooncogenes MYC and 
STAT3 and loss of or mutations in the tumor suppressors $C D K N 2 A / B-T P 53$ are genomic features of PTCL-GATA3, accompanying y higher levels of $M Y C$ transcripts and enrichment of MYC-target genes. ${ }^{17}$ Manso et al. also reported MYC expression to be positively correlated with GATA3 expression in PTCL, NOS, based on IHC. ${ }^{42}$

\section{Impact of genomic abnormalities on prognosis of patients with PTCL, NOS}

Heavican et al. also reported that $C D K N 2 A$ deletion is associated with poor prognosis in both PTCL, NOS and PTCL-GATA3 subgroups. ${ }^{17}$ Watatani et al. performed targeted sequencing in 140 genes in 142 PTCL, NOS cases and identified a distinct subgroup after excluding nPTCL-Tfh associated with poor prognosis. ${ }^{18}$ Those included: TP53/ CDKN2A mutations and deletions, accompanied by chromosomal instability as well as mutations associated with immune escape (such as HLA-A and HLA-B, CIITA, CD274 and $C D 58)$ and transcription $(I K Z F 2){ }^{18}$

\section{Recurrently mutant genes in PTCL, NOS}

\section{Mutations in genes encoding epigenetic regulators}

Mutations in genes encoding DNA modifying enzymes such as TET2 and DNMT3A occur in numerous blood cancers. These mutations were initially identified in myeloid malignancies ${ }^{43,44}$ and subsequently found in lymphoid malignancies including PTCL, NOS. ${ }^{24}$ Notably, these mutations are believed to sometimes occur as a first hit in tumorigenesis in hematopoietic stem/progenitor cells that undergo differentiation into various blood cell subtypes. ${ }^{28}$ They are seen in various lineages of blood cells as wells as tumor cells in PTCL patients, ${ }^{24-27}$ and recur in elderly individuals with clonal hematopoiesis. ${ }^{45,46}$ TET2 mutations are as frequent as $58 \% \%^{32}-98 \%{ }^{18}$ in nPTCL-Tfh but are less frequent but occur in $24 \%$ of PTCL, NOS without Tfh features. ${ }^{32}$ Watatani et al. reported that $21 \%$ of PTCL, NOS with TP53/CDKN2A alterations also harbored TET2 mutations, while TET2 mutations were seen in only $2 \%$ of other PTCL, NOS cases. ${ }^{18}$

\section{The p.Gly17Val RHOA mutation}

Recurrent hotspot RHOA mutations that convert Glycine to Valine at amino acid 17 occur in up to $70 \%$ of AITL ${ }^{33,47}$ and are broadly found in "AITL and other nodal lymphomas of Tfh cell origin," including both nPTCL-Tfh ${ }^{18,33,34}$ and FTCL. ${ }^{35,48}$ These mutations do not occur in PTCL, NOS excluding nPTCL-Tfh. Fujisawa et al. found that p.Gly17Val RHOA mutant protein binds to VAV1 and facilitates its phosphorylation by Src family kinases, hyperactivating TCR signaling. ${ }^{49}$ Zang et al., however, have reported that expression of p.GlyVal RHOA mutant increases phosphorylation of FoxO1, ${ }^{50}$ promoting its translocation from the nucleus to the cytoplasm and subsequent inactivation by proteasomal degradation.

\section{Mutations in genes functioning in TCR signaling}

TCR signaling increases proliferation and survival of normal T cells. Intriguingly, PTCL tumor cells utilize TCR signaling pathways that promote survival and proliferation of normal $\mathrm{T}$ cells. Activating mutations in genes encoding TCR signaling molecules are recurrent in various PTCLs, including PTCL, NOS, although mutation frequency varies among disease subtypes. Due to the limited mutational data relevant to TCR signaling in PTCL, NOS, the mutational sites and consequences of mutations referring to findings in other PTCLs are included.

\section{CD28 mutations}

CD28 is a costimulatory molecule for TCR activation. CD28 mutations occur in $0 \%{ }^{34}-10 \%{ }^{18}$ of nPTCL-Tfh and $19 \%$ of PTCL, NOS with TP53/CDKN2A alterations, but are rare in other forms of PTCL, NOS. ${ }^{18}$ CD28 mutations recurrently occur at p.Asp124 and p.Thr195. ${ }^{34,51}$ The p.Asp124 mutant exhibits higher affinity for the ligands CD80 and CD86, ${ }^{51}$ while the p.Thr 195 mutant facilitates interaction with intracellular adaptor proteins, such as GADS/GRAP2 and GRB2. ${ }^{51,52}$

\section{VAV1 mutations and translocations}

Vav guanine nucleotide exchange factor 1(VAVI) encodes a guanine exchange factor (GEF) that facilitates GDP/GTP exchange and exhibits $\mathrm{SH} 2$ and $\mathrm{SH} 3$ domains at the $\mathrm{C}$ terminus. ${ }^{53}$ Upon TCR stimulation, VAV1 is phosphorylated by the Src family kinases LCK and FYN at Tyrs 142, 160, and 174 in the acidic (Ac) domain. Phosphorylated VAV1 then mediates TCR signaling in a GEF-dependent manner by activating small GTPases such as Rac1 and also in a GEFindependent manner by forming an active complex with $\mathrm{SH} 2$ domain-containing leukocyte protein of 76kDa (SLP76) and phospholipase C, gamma 1 (PLCG1). ${ }^{53}$ VAV1 mutations occur across various PTCL subtypes. Among them, $12 \%{ }^{18}$ $0 \%{ }^{34}$ in nPTCL-Tfh; $5 \%$ in PTCL, NOS with TP53/CDKN2A alterations; ${ }^{18}$ and none in other PTCL, NOS subtypes. ${ }^{18}$ Also observed are missense mutations at several hotspots in the Ac (Glu175), pleckstrin homology (PH, Lys404), zinc-finger (ZF, Glu556) and SH3 (Arg798 and Arg822) domains, as well as focal in-frame deletions in the Ac domain $(\Delta 165$ 174). Deletion $(\Delta 778-786)$ of the $\mathrm{C}$-terminal SH3 domain ${ }^{54}$ by alternative splicing or by fusion with other partner genes resulting from chromosomal translocations is also recurrently seen. ${ }^{49,54,55}$ Alterations in either the Ac or SH3-SH2-SH3 domains enhance TCR signaling by disrupting autoinhibitory mechanisms. ${ }^{49,54}$ Potential changes in downstream signaling due to either PH or ZF domain mutations have not yet been described. The function of VAV1 mutations in in vivo lymphomagenesis is described below (See "VI. Mouse models of PTCL, NOS").

\section{PLCG1 mutations}

PLCG1 mutations occur in several PTCL, NOS subtypes: $4 \%{ }^{18}-35 \%{ }^{34}$ in nPTCL-Tfh; $24 \%$ in PTCL, NOS with TP53/ CDKN2A alterations; ${ }^{18}$ and $5 \%$ in other forms of PTCL, NOS. ${ }^{18}$ PLCG1 mutations accumulate in several functional motifs, including the PI-PLC, SH2, SH3, and $\mathrm{C} 2$ domains. 
PLCG1 mutants reportedly hyperactivate TCR signaling in vitro, ${ }^{34}$ while their consequences in vivo remain unclear.

\section{ITK-SYK translocation}

A translocation $\mathrm{t}(5 ; 9)(\mathrm{q} 33 ; \mathrm{q} 22)$ was recurrently found in PTCL. ${ }^{56}$ At the breakpoint N-terminal PH and TH domains of ITK fuse to the kinase domain of SYK. ${ }^{56}$ Notbaly, ITK$S Y K$ was found in $3 / 5(60 \%)^{56}-4 / 22(18 \%)^{57}$ of FTCL cases. ITK-FER fusion was also found in a case of PTCL. ${ }^{55}$ The $I T K-S Y K$ fusion gene induces antigen-independent phosphorylation of TCR-related molecules, leading to hyperactivation of TCR signaling. ${ }^{58}$ The in vivo function of ITK-SYK is described below (See "VI. Mouse models of PTCL, NOS").

\section{Mutations in genes related to immune escape}

As noted, recurrent mutations in genes related to immune escape, including $H L A-A, H L A-B, C I I T A, C D 274$, and CD58 genes are observed in PTCL, NOS, especially in the subgroup with TP53/CDKN2A mutations. ${ }^{18}$ It is presumed that among tumor cells exhibiting genomic instability, clones that acquire mechanisms allowing immune escape to survive.

\section{MOUSE MODELS OF PTCL, NOS}

Mouse models that recapitulate genomic abnormalities observed in human cancers are essential for understanding pathological mechanisms and testing new treatment strategies in vivo. As noted, activating VAV1 mutations are seen in various PTCL subtypes, occasionally together with TP53 genomic alterations in the PTCL-GATA3 subgroup. ${ }^{17}$ Fukumoto et al. created transgenic mice expressing two VAV1 mutants: an in-frame deletion mutant in the Ac domain and a fusion gene with STAP2, both cloned from human PTCL under the human CD2 promoter (VAV1-tg mice) ${ }^{59}$ (Figure 1A). Although no tumors were seen in VAV1-tg mice for up to a year, mature T-cell lymphomas mimicking human PTCL-GATA3 were observed when VAV1-Tg mice were crossed with Trp53 knockout mice. Lymphoma cells expressing mutant VAV1 gene on a Trp53-null background exhibited Th2 characteristics, including high expression of Gata3 and Ccr4 proteins ${ }^{59}$ (Figure 1B). Notably, these phenotypes differed completely from our previous report that enrichment of a TFH signature was evident in lymphoma cells from AITL model mice expressing G17V RHOA on a Tet2-null background ${ }^{59}$ (Figure 1B), although VAV1 activation was also observed in these lymphoma cells. ${ }^{60}$ Presumably, coexisting mutations (Tet2-null vs Trp53-null) account for phenotypic differences between these mice..$^{59,60}$ Enrichment of multiple Myc-related pathways based on GEP analysis together with determination of focal somatic copy number alterations (SCNAs) involving the $M y c$ locus were also recurrently observed ${ }^{59}$ (Figure 1C), as seen in human PTCLGATA3. ${ }^{17}$ Inhibition of Myc signaling by a bromodomain inhibitor prolonged survival of nude mice transplanted with tumors simultaneously harboring VAV1 mutation and P53 deletion $^{59}$ (Figure 1D).

As mentioned above, ITK-SYK fusion gene is recurrently observed in PTCL, especially in FTCL. ${ }^{6,57}$ Conditional expression of ITK-SYK fusion gene in the T-cell lineage leads to aggressive PTCL-like diseases in mice. ${ }^{58}$ Tumor cells of individual mice were either CD4-positive, CD8-positive, or a mix of CD4-positive and CD8-positive. Transplantation of 5-fluorouracil-pretreated bone marrow cells with retroviral transduction of ITK-SYK cDNA also induced a T-cell lymphoproliferative disease in the recipient mice, accompanying systemic inflammation. ${ }^{61}$ A SYK kinase inhibitor alleviated the disease progression in mice. ${ }^{61}$

\section{TRANSLATION OF MOLECULAR ANALYSIS TO THE BED-SIDE MANAGEMENT OF PTCL, NOS}

Translational researchers are actively assessing GEP and genomic abnormalities in cancer in order to apply them to clinical practice. "Precision medicine", also known as "optimized" or "personalized" medicine, is a widely used term that refers to creating diagnostic or prognostic algorism and treatment regimes based on the nature of a patient's tumor.

\section{A "precision medicine approach" to utilize GEP information relevant to PTCL, NOS}

Attempts to stratify heterogeneous populations of blood cancers using GEPs began with analysis of diffuse large B-cell lymphoma (DLBCL) and employed the largest cohort of malignant lymphomas. In 2000, Alizadeh et al. reported molecular classifications of the DLBCL subtypes of activated B-cell (ABC)-type, germinal center B-cell (GCB)-type and unclassifiable, ${ }^{62}$ based on GEPs using DNA microarrays. That analysis was reproduced by $\mathrm{IHC}^{63}$ and digital gene expression analysis. ${ }^{64}$ Patients with ABC-type DLBCL reportedly had a worse prognosis than those with GCB type. Therefore, DLBCL classification in this manner is essential, especially when setting up clinical trials. According to similarity of biological nature of tumors, clinical trials involving both nPTCL-Tfh and AITL are under way. It may also be appropriate to treat PTCL-GATA3 and PTCL-TBX21 subgroups separately in clinical trials due to their different prognosis. Mogamulizumab, an antibody drug targeting CCR4, has been used to treat PTCL after diagnosis of CCR4 positivity. Its effectiveness is expected particularly in the PTCLGATA3 subtype, considering that CCR4 expression is reportedly correlated with GATA3 expression, ${ }^{39}$ as noted above.

\section{A "precision medicine approach" to utilize genomic information relevant to PTCL, NOS}

With improvement of next-generation sequencing technologies, sequencing costs have decreased significantly over time based on a pattern known as Moore's Law, and now sequence analysis is accessible in clinical settings. In the midst of dramatic changes in cancer genome analysis, in 2015 US President Obama advocated a "Precision medicine initiative" in his State of the Union address, claiming that the US would invest a large portion of its national budget to encourage precision approaches to cancer based on genomic 

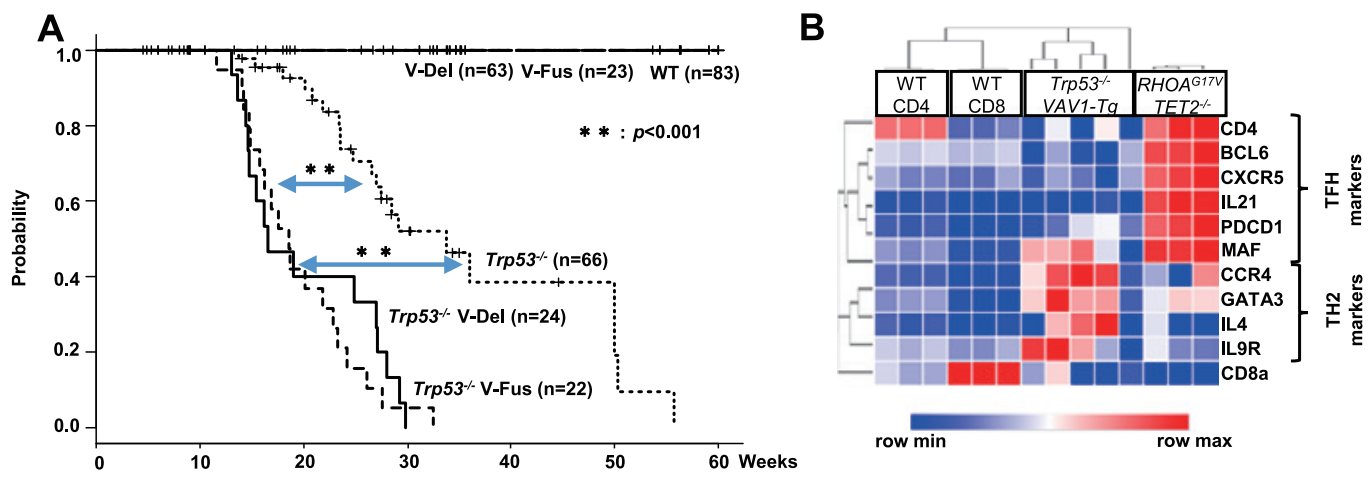

C (i)

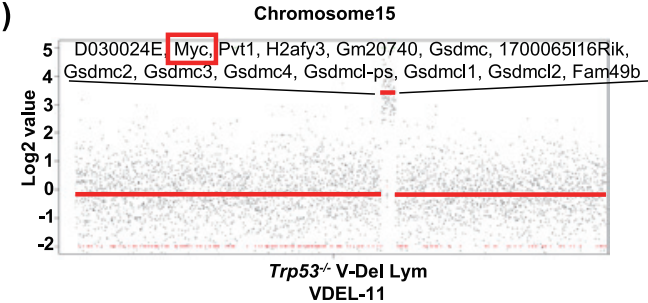

(ii)

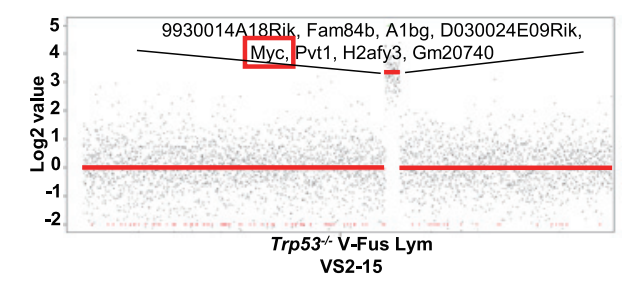

D (i)

Overall survival

(ii)

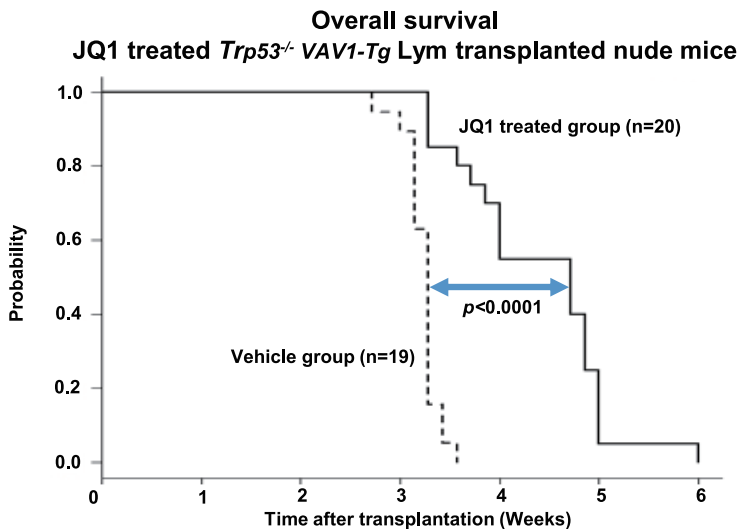

Fig. 1. VAV1-mutant expression in Trp53-null background induces development of T-lymphoblastic lymphomas (LBL) and lymphomas mimicking human PTCL-GATA3 in mice (Cited from figures in (59)).

$\boldsymbol{A}$. Overall survival of mice with each genotype. V-Del, a human VAV1 mutant (p.165_174del) expressing mice; V-Fus, a VAV1STAP2 fusion gene expressing mice; WT, wild-type mice.

$\boldsymbol{B}$. Supervised clustering of gene expression profiles for tumors cells expressing VAV1-mutant in Trp53-null background, those expressing RHOA-mutant in Trp53-null background, and $\mathrm{CD}^{+}$or $\mathrm{CD}^{+}{ }^{+} \mathrm{T}$ cells from wild-type mice. WT, wild type; TFH, T follicular helper cell; TH2, helper T-cell, type2.

C. Amplifications of Myc locus in tumor cells expressing VAV1-mutant in Trp53-null background.

D. The effect of JQ1, a bromodomain inhibitor treatment in nude mice transplanted with tumor cells expressing VAV1-mutant expression in Trp53-null background.

information. Remarkably, such precision medicine for patients with solid tumors is under way: Oncogene panel testing began in November 2017 in the US. And in Japan, two Oncogene panel tests have been approved since December of 2018 and are covered by health insurance. However, relevant to hematologic malignancies including PTCL, Oncogene panel testing is currently not available (as of October, 2020), although individual genome testing has been widely used in clinical practice for a long time.

To date, PTCL, NOS is diagnosed by combining histopathological findings such as tumor cell morphology and immunohistochemistry with clinical features. However, it is sometimes difficult to distinguish PTCL, NOS from other lymphomas or even from reactive lymphadenopathy.
Addition of oncogene panel testing to current methods would enable more accurate "diagnosis" of PTCL, NOS among subtypes and discriminate between benign and malignant tumors.

As noted, retrospective studies show that prognosis of PTCL patients is stratified based on genomic abnormalities. ${ }^{17,18}$ These results must be confirmed by prospective analysis but may provide guidance for treatment choices. For patients in the poor prognosis group who do not benefit from conventional treatments, several options including clinical trials with novel drug combinations or consolidation therapies with allogenic transplantation might be recommended.

Therapeutic approaches targeting mutant proteins or downstream signaling factors are under active investigation. 
Loss-of-function TET2 mutations seen in hematologic cancers promote DNA hypermethylation. One retrospective study reported that $9(75 \%)$ of 12 AITL patients whose tumors had TET2 mutations responded to azacytidine, a DNA methylation inhibitor. ${ }^{65}$ Because TET2 mutations are also seen in nPTCL-Tfh and other forms of PTCL, NOS, inhibition of DNA methylation is could be a promising approach in these cases as well. The p.Gly17Val RHOA mutant also provides a potential target in nPTCL-Tfh and AITL. Enhanced VAV1 phosphorylation by Src family kinases as a consequence of p.Gly17Val RHOA mutation is blocked by dasatinib, a multikinase inhibitor, ${ }^{49}$ and dasatinib prolongs survival of AITL model mice. ${ }^{60}$ A phase 1 clinical trial showed that 4 of 5 relapsed/refractory AITL patients treated with dasatinib achieved a partial response. ${ }^{60}$ The PI3K-Akt-mTOR pathway is also downstream of the p.Gly17Val RHOA mutant. ${ }^{50,66,67} \mathrm{Ng}$ et al.$^{67}$ and Cortes et al. ${ }^{66}$ reported that everolimus, an mTOR inhibitor, and duvelisib, a selective small molecule PI3K $\delta / \gamma$ inhibitor, slowed tumor progression in AITL model mice. Witzig et al. reported that everolimus had antitumor activity against T-cell lymphomas in vivo, though only one AITL patient was included in that study. ${ }^{68}$ A phase II study of everolimus plus CHOP for newly diagnosed PTCL demonstrated that all 3 AITL patients achieved a complete response (CR) ${ }^{69}$ Horwitz et al. reported antitumor activity of duvelisib against T-cell lymphomas in a phase 1 study. ${ }^{70,71}$ Among 3 AITL patients in that study, CR and partial response was achieved by each patient. ${ }^{70}$ Ghione et al. reported that relapsed/refractory patients with PTCL who showed either AITL or nPTCL-Tfh phenotypes had higher response rate to histone deacetylase inhibitors (HDACi), including romidepsine and belinostat, than those without. ${ }^{72}$ Gene mutations were examined in some samples included in this study. Intriguingly, patients responsive to HDACi more frequently exhibited AITL-specific mutational profiles (mutations in $T E T 2$, and/or DNMT3A, and/or RHOA $)^{33}$ than those without, although the relationship between clinical efficacy and mutational profiles must be confirmed in a larger cohort. $^{72}$

\section{FUTURE PERSPECTIVES}

Investigators continue to identify new PTCL, NOS subgroups with distinct diagnostic and prognostic features. If prognosis under current therapeutic management can be properly predicted, it could be useful to avoid conventional strategies and proceed to enrollment into clinical trials. Basic researchers are rigorously conducting analysis required to target genomic abnormalities in PTCL, NOS. A "precision medicine approach" to PTCL, NOS will identify the correct treatment choice once a direct therapeutic target is identified, or if results of genome/GEP testing can stratify patients for whom a treatment is either effective or ineffective. These efforts should lead to better management of PTCL, NOS patients in the near future.

\section{ADDITIONAL INFORMATION}

This work was supported by Grants-in-Aid for Scientific Research (KAKENHI: 18H02834 [M.S.-Y.]) from the Ministry of Education, Culture, Sports, and Science of Japan; and an AMED under Grant Numbers JP20ck0106544h and JP19lm0203023 (M.S.-Y.).

\section{CONFLICT OF INTEREST}

M.S.-Y. received funds from Bristol Myers Squibb, Otsuka Pharmaceuticals, and Eisai Co.. S.C. received funds from Thyas, Takeda Pharmaceutical, Chugai Pharmaceutical, Kyowa-Kirin, Ono Pharmaceutical, Sanofi, and Astellas Pharmaceutical. The remaining authors declare no competing financial interests.

\section{REFERENCES}

1 Vose J, Armitage J, Weisenburger D; International T-Cell Lymphoma Project. International peripheral T-cell and natural killer/T-cell lymphoma study: pathology findings and clinical outcomes. J Clin Oncol. 2008; 26 : 4124-4130.

2 Swerdllow S, Campo E, Harris NL, et al. WHO Classification of Tumours of Haematopoietic and Lymphoid Tissues. IARC. 2017.

3 Iqbal J, Wright G, Wang C, et al. Gene expression signatures delineate biological and prognostic subgroups in peripheral T-cell lymphoma. Blood. 2014; $123:$ 2915-2923.

4 de Leval L, Rickman DS, Thielen C, et al. The gene expression profile of nodal peripheral T-cell lymphoma demonstrates a molecular link between angioimmunoblastic T-cell lymphoma (AITL) and follicular helper T (TFH) cells. Blood. 2007; 109 : 4952-4963.

5 Piccaluga PP, Agostinelli C, Califano A, et al. Gene expression analysis of peripheral $\mathrm{T}$ cell lymphoma, unspecified, reveals distinct profiles and new potential therapeutic targets. J Clin Invest. 2007; $117: 823-834$

6 Iqbal J, Weisenburger DD, Greiner TC, et al. Molecular signatures to improve diagnosis in peripheral T-cell lymphoma and prognostication in angioimmunoblastic T-cell lymphoma. Blood. 2010; 115 : 1026-1036.

7 Huang Y, de Reyniès A, de Leval L, et al. Gene expression profiling identifies emerging oncogenic pathways operating in extranodal NK/T-cell lymphoma, nasal type. Blood. 2010; 115 : 1226-1237.

8 Iqbal J, Weisenburger DD, Chowdhury A, et al. Natural killer cell lymphoma shares strikingly similar molecular features with a group of non-hepatosplenic $\gamma \delta$ T-cell lymphoma and is highly sensitive to a novel aurora kinase A inhibitor in vitro. Leukemia. 2011; $25: 348-358$.

9 Morris SW, Kirstein MN, Valentine MB, et al. Fusion of a kinase gene, ALK, to a nucleolar protein gene, NPM, in nonHodgkin's lymphoma. Science. 1995; 267 : 316-317.

10 Chiarle R, Simmons WJ, Cai H, et al. Stat 3 is required for ALK-mediated lymphomagenesis and provides a possible therapeutic target. Nat Med. 2005; 11 : 623-629. 
11 Piccaluga PP, Fuligni F, De Leo A, et al. Molecular profiling improves classification and prognostication of nodal peripheral T-cell lymphomas: results of a phase III diagnostic accuracy study. J Clin Oncol. 2013; $31:$ 3019-3025.

12 Piccaluga PP, Agostinelli C, Califano A, et al. Gene expression analysis of angioimmunoblastic lymphoma indicates derivation from $\mathrm{T}$ follicular helper cells and vascular endothelial growth factor deregulation. Cancer Res. 2007; 67 : 10703-10710.

13 Asano N, Suzuki R, Kagami Y, et al. Clinicopathologic and prognostic significance of cytotoxic molecule expression in nodal peripheral T-cell lymphoma, unspecified. Am J Surg Pathol. 2005; 29 : 1284-1293.

14 Went P, Agostinelli C, Gallamini A, et al. Marker expression in peripheral T-cell lymphoma: a proposed clinical-pathologic prognostic score. J Clin Oncol. 2006; 24 : 2472-2479.

15 McKinney M, Moffitt AB, Gaulard P, et al. The Genetic Basis of Hepatosplenic T-cell Lymphoma. Cancer Discov. 2017; 7 : 369-379.

16 Moffitt AB, Ondrejka SL, McKinney M, et al. Enteropathyassociated $\mathrm{T}$ cell lymphoma subtypes are characterized by loss of function of SETD2. J Exp Med. 2017; 214 : 1371-1386.

17 Heavican TB, Bouska A, Yu J, et al. Genetic drivers of oncogenic pathways in molecular subgroups of peripheral T-cell lymphoma. Blood. 2019; 133 : 1664-1676.

18 Watatani Y, Sato Y, Miyoshi H, et al. Molecular heterogeneity in peripheral T-cell lymphoma, not otherwise specified revealed by comprehensive genetic profiling. Leukemia. 2019; 33 : 2867-2883.

19 Gayden T, Sepulveda FE, Khuong-Quang DA, et al. Germline HAVCR2 mutations altering TIM-3 characterize subcutaneous panniculitis-like T cell lymphomas with hemophagocytic lymphohistiocytic syndrome. Nat Genet. 2018; 50 : 1650-1657.

20 Polprasert C, Takeuchi Y, Kakiuchi N, et al. Frequent germline mutations of HAVCR2 in sporadic subcutaneous panniculitislike T-cell lymphoma. Blood Adv. 2019; 3 : 588-595.

21 Nakagawa M, Nakagawa-Oshiro A, Karnan S, et al. Array comparative genomic hybridization analysis of PTCL-U reveals a distinct subgroup with genetic alterations similar to lymphomatype adult T-cell leukemia/lymphoma. Clin Cancer Res. 2009; $15: 30-38$.

22 Kataoka K, Nagata Y, Kitanaka A, et al. Integrated molecular analysis of adult $\mathrm{T}$ cell leukemia/lymphoma. Nat Genet. 2015; $47: 1304-1315$.

23 de Leval L, Gaulard P. Cellular origin of T-cell lymphomas. Blood. 2014; 123 : 2909-2910.

24 Quivoron C, Couronné L, Della Valle V, et al. TET2 inactivation results in pleiotropic hematopoietic abnormalities in mouse and is a recurrent event during human lymphomagenesis. Cancer Cell. 2011; $20: 25-38$.

25 Couronné L, Bastard C, Bernard OA. TET2 and DNMT3A mutations in human T-cell lymphoma. N Engl J Med. 2012; 366 : 95-96.

26 Nguyen TB, Sakata-Yanagimoto M, Asabe Y, et al. Identification of cell-type-specific mutations in nodal T-cell lymphomas. Blood Cancer J. 2017; 7 : e516.

27 Nguyen TB, Sakata-Yanagimoto M, Nakamoto-Matsubara R, et al. Double somatic mosaic mutations in TET2 and
DNMT3A-origin of peripheral T cell lymphoma in a case. Ann Hematol. 2015; 94 : 1221-1223.

28 Sakata-Yanagimoto M. Multistep tumorigenesis in peripheral T cell lymphoma. Int J Hematol. 2015; 102 : 523-527.

29 Kluin PM. Origin and migration of follicular lymphoma cells. Haematologica. 2013; 98 : 1331-1333.

30 Lieber MR. Mechanisms of human lymphoid chromosomal translocations. Nat Rev Cancer. 2016; $16: 387-398$.

31 Rodríguez-Pinilla SM, Atienza L, Murillo C, et al. Peripheral T-cell lymphoma with follicular T-cell markers. Am J Surg Pathol. 2008; 32 : 1787-1799.

32 Lemonnier F, Couronné L, Parrens M, et al. Recurrent TET2 mutations in peripheral T-cell lymphomas correlate with TFHlike features and adverse clinical parameters. Blood. 2012; 120 : 1466-1469.

33 Sakata-Yanagimoto M, Enami T, Yoshida K, et al. Somatic RHOA mutation in angioimmunoblastic $\mathrm{T}$ cell lymphoma. Nat Genet. 2014; $46:$ 171-175.

34 Vallois D, Dobay MPD, Morin RD, et al. Activating mutations in genes related to TCR signaling in angioimmunoblastic and other follicular helper T-cell-derived lymphomas. Blood. 2016; $128: 1490-1502$.

35 Dobay MP, Lemonnier F, Missiaglia E, et al. Integrative clinicopathological and molecular analyses of angioimmunoblastic T-cell lymphoma and other nodal lymphomas of follicular helper T-cell origin. Haematologica. 2017; 102 : e148-e151.

36 Wang T, Feldman AL, Wada DA, et al. GATA-3 expression identifies a high-risk subset of PTCL, NOS with distinct molecular and clinical features. Blood. 2014; 123 : 3007-3015.

37 Zhang Y, Zhang Y, Gu W, Sun B. TH1/TH2 cell differentiation and molecular signals. Adv Exp Med Biol. 2014; 841 : 15-44.

38 Crotty S. T follicular helper cell differentiation, function, and roles in disease. Immunity. 2014; $41: 529-542$.

39 Amador C, Greiner TC, Heavican TB, et al. Reproducing the molecular subclassification of peripheral T-cell lymphomaNOS by immunohistochemistry. Blood. 2019; 134 : 2159-2170.

40 Sugio T, Miyawaki K, Kato K, et al. Microenvironmental immune cell signatures dictate clinical outcomes for PTCLNOS. Blood Adv. 2018; 2 : 2242-2252.

41 Swerdlow SH, Campo E, Pileri SA, et al. The 2016 revision of the World Health Organization classification of lymphoid neoplasms. Blood. 2016; 127 : 2375-2390.

42 Manso R, Bellas C, Martín-Acosta P, et al. C-MYC is related to GATA3 expression and associated with poor prognosis in nodal peripheral T-cell lymphomas. Haematologica. 2016; 101 : e336-e338.

43 Ley TJ, Ding L, Walter MJ, et al. DNMT3A mutations in acute myeloid leukemia. N Engl J Med. 2010; 363 : 2424-2433.

44 Langemeijer SMC, Kuiper RP, Berends M, et al. Acquired mutations in TET2 are common in myelodysplastic syndromes. Nat Genet. 2009; $41: 838-842$.

45 Genovese G, Kähler AK, Handsaker RE, et al. Clonal hematopoiesis and blood-cancer risk inferred from blood DNA sequence. N Engl J Med. 2014; 371 : 2477-2487.

46 Jaiswal S, Fontanillas P, Flannick J, et al. Age-related clonal hematopoiesis associated with adverse outcomes. N Engl J Med. 2014; 371 : 2488-2498. 
47 Palomero T, Couronné L, Khiabanian H, et al. Recurrent mutations in epigenetic regulators, RHOA and FYN kinase in peripheral T cell lymphomas. Nat Genet. 2014; 46 : 166-170.

48 Miyoshi H, Sakata-Yanagimoto M, Shimono J, et al. RHOA mutation in follicular T-cell lymphoma: Clinicopathological analysis of 16 cases. Pathol Int. 2020; $70: 653-660$.

49 Fujisawa M, Sakata-Yanagimoto M, Nishizawa S, et al. Activation of RHOA-VAV1 signaling in angioimmunoblastic T-cell lymphoma. Leukemia. 2018; $32: 694-702$.

50 Zang S, Li J, Yang H, et al. Mutations in 5-methylcytosine oxidase TET2 and RhoA cooperatively disrupt $\mathrm{T}$ cell homeostasis. J Clin Invest. 2017; 127 : 2998-3012.

51 Rohr J, Guo S, Huo J, et al. Recurrent activating mutations of CD28 in peripheral T-cell lymphomas. Leukemia. 2016; 30 : 1062-1070.

52 Lee SH, Kim JS, Kim J, et al. A highly recurrent novel missense mutation in CD28 among angioimmunoblastic T-cell lymphoma patients. Haematologica. 2015; 100 : e505-e507.

53 Katzav S. Vav1: A Dr. Jekyll and Mr. Hyde protein - good for the hematopoietic system, bad for cancer. Oncotarget. 2015; 6 : 28731-28742.

54 Abate F, da Silva-Almeida AC, Zairis S, et al. Activating mutations and translocations in the guanine exchange factor VAV1 in peripheral T-cell lymphomas. Proc Natl Acad Sci USA. 2017; 114 : 764-769.

55 Boddicker RL, Razidlo GL, Dasari S, et al. Integrated mate-pair and RNA sequencing identifies novel, targetable gene fusions in peripheral T-cell lymphoma. Blood. 2016; 128 : 1234-1245.

56 Streubel B, Vinatzer U, Willheim M, Raderer M, Chott A. Novel $\mathrm{t}(5 ; 9)(\mathrm{q} 33 ; \mathrm{q} 22)$ fuses ITK to SYK in unspecified peripheral T-cell lymphoma. Leukemia. 2006; 20 : 313-318.

57 Huang Y, Moreau A, Dupuis J, et al. Peripheral T-cell lymphomas with a follicular growth pattern are derived from follicular helper T cells (TFH) and may show overlapping features with angioimmunoblastic T-cell lymphomas. Am J Surg Pathol. 2009; $33: 682-690$.

58 Pechloff K, Holch J, Ferch U, et al. The fusion kinase ITK-SYK mimics a $\mathrm{T}$ cell receptor signal and drives oncogenesis in conditional mouse models of peripheral T cell lymphoma. J Exp Med. 2010; 207 : 1031-1044.

59 Fukumoto K, Sakata-Yanagimoto M, Fujisawa M, et al. VAV1 mutations contribute to development of T-cell neoplasms in mice. Blood. 2020; 136 : 3018-3032.

60 Nguyen TB, Sakata-Yanagimoto M, Fujisawa M, et al. Dasatinib is an effective treatment for angioimmunoblastic T-cell lymphoma. Cancer Res. 2020; $80:$ 1875-1884.
61 Dierks C, Adrian F, Fisch P, et al. The ITK-SYK fusion oncogene induces a T-cell lymphoproliferative disease in mice mimicking human disease. Cancer Res. 2010; 70 : 6193-6204.

62 Alizadeh AA, Eisen MB, Davis RE, et al. Distinct types of diffuse large B-cell lymphoma identified by gene expression profiling. Nature. 2000; $403:$ 503-511.

63 Hans CP, Weisenburger DD, Greiner TC, et al. Confirmation of the molecular classification of diffuse large B-cell lymphoma by immunohistochemistry using a tissue microarray. Blood. 2004; $103: 275-282$

64 Scott DW, Mottok A, Ennishi D, et al. Prognostic Significance of Diffuse Large B-Cell Lymphoma Cell of Origin Determined by Digital Gene Expression in Formalin-Fixed ParaffinEmbedded Tissue Biopsies. J Clin Oncol. 2015; 33 : 2848-2856.

65 Lemonnier F, Dupuis J, Sujobert P, et al. Treatment with 5-azacytidine induces a sustained response in patients with angioimmunoblastic T-cell lymphoma. Blood. 2018; 132 : 2305-2309.

66 Cortes JR, Ambesi-Impiombato A, Couronné L, et al. RHOA G17V Induces T Follicular Helper Cell Specification and Promotes Lymphomagenesis. Cancer Cell. 2018; 33 : 259-273. e7.

$67 \mathrm{Ng} \mathrm{SY}$, Brown L, Stevenson K, et al. RhoA G17V is sufficient to induce autoimmunity and promotes T-cell lymphomagenesis in mice. Blood. 2018; 132 : 935-947.

68 Witzig TE, Reeder C, Han JJ, et al. The mTORC1 inhibitor everolimus has antitumor activity in vitro and produces tumor responses in patients with relapsed T-cell lymphoma. Blood. $2015 ; 126: 328-335$

69 Kim SJ, Shin DY, Kim JS, et al. A phase II study of everolimus (RAD001), an mTOR inhibitor plus CHOP for newly diagnosed peripheral T-cell lymphomas. Ann Oncol. 2016; 27 : 712-718.

70 Horwitz SM, Koch R, Porcu P, et al. Activity of the PI3K- $\delta, \gamma$ inhibitor duvelisib in a phase 1 trial and preclinical models of T-cell lymphoma. Blood. 2018; 131 : 888-898.

71 Flinn IW, O'Brien S, Kahl B, et al. Duvelisib, a novel oral dual inhibitor of PI3K- $\delta, \gamma$, is clinically active in advanced hematologic malignancies. Blood. 2018; $131: 877-887$

72 Ghione P, Faruque P, Mehta-Shah N, et al. T follicular helper phenotype predicts response to histone deacetylase inhibitors in relapsed/refractory peripheral T-cell lymphoma. Blood Adv. $2020 ; 4$ : 4640-4647. 